\title{
Congestion management optimization in electric transmission system
}

\author{
E. Semshchikov \\ School of Engineering, College of Science and Engineering \\ University of Tasmania \\ Hobart, Australia \\ evgenii.semshchikov@utas.edu.au
}

\author{
M. Negnevitsky \\ School of Engineering, College of Science and Engineering \\ University of Tasmania \\ Hobart, Australia \\ michael.negnevitksy@utas.edu.au
}

\begin{abstract}
Congestion management in electric transmission systems is one of the most important challenges for power systems with high penetration of renewable energy. System congestion occurs when the desired power flow cannot be transmitted through the network without violating system operating limits. In order to prevent severe system damage, a significant number of congestion management methods have been developed, including nodal pricing, load shedding, curtailment of renewable energy generation, generator rescheduling, optimal transmission switching, etc. Most of these methods, however, do not comply with the optimal operation of conventional power plants subjected to dynamic constraints (manoeuvrability, start-up and shut down times, etc.). In this paper, the rescheduling generation (or re-dispatch optimization) problem is solved using a modified particle swarm optimization (PSO) algorithm which accounts for start up as well as shut down times, and the manoeuvrability of conventional power plants.
\end{abstract}

Index Terms--Congestion management, Particle swarm optimization, Transmission system

\section{INTRODUCTION}

In order to promote competition in the electricity generation industry, many countries continue to replace traditionally regulated monopolistic markets comprised of vertically integrated utilities with competitive power markets. Although the competitive model contributes to an overall increase in operational and financial efficiency, it entails several challenges. Generating companies are forced to install power plants in areas with the highest rate of return. Consequently, low-cost power generation is concentrated in a particular region and is favoured by both close and distant consumers [1].

In counties with renewable energy policy targets where electricity companies are mandated to provide a particular percentage of generated power from renewable energy sources the situation can be further aggravated. In Germany, for example, low-cost energy is mainly generated in the north where the coal transportation cost is minimal (coal is supplied to the north of the country via the North and Baltic Seas), the wind is strong and offshore wind farms are installed [2].

Transmission of electrical energy is bounded by system operating limits such as thermal, stability and voltage. In [3], available transfer capability is obtained using the linear method based on the power transmission distribution factor (PTDF) matrix. Prediction and correction techniques, for example, the continuation power flow (CPF) method are used [4]. A method generally adopted in the power industry to compute total transfer capability is the repetitive power flow (RPF)[5].

When an electricity transmission system is unable to transmit all the desired electrical power without violating system operating limits, it is said to be congested. Congestion also occurs due to sudden increase on load, loss of generation and other equipment failures [6]. It can harm power quality, cause equipment damage and even lead to widespread blackouts. Congestion management is an approach whose goal is to control the transmission system, so that all power flows remain within operational limits. In [7], authors provide a classification of congestion management methods. Methods can be either technical or non-technical, and pricing or nonpricing. Technical methods such as curtailment of the congested transaction, changing transformer taps, operating FACTS devices, etc. are all non-pricing [8]. Optimal topological configuration (or the optimal transmission switching (OTS) problem) was discussed in [9]. Authors in [10], applied the genetic algorithm to solve OTS problems for the Italian power system. Nodal pricing, zonal pricing and market split are examples of non-technical pricing methods $[11,12]$.

The most commonly used congestion management method is generator rescheduling (GR) or re-dispatch (RD), where in order to clear congestion, the Independent System Operator (ISO) may request some power plants to increase generation whereas others would be required to decrease it [13]. As result, the ISO incurs costs, which will be eventually included in consumer electricity bills. Therefore, the re-dispatch optimization problem, aimed at minimizing such costs, is of 
paramount importance. Many optimization techniques have been applied to solve problems of the power system industry. Traditional optimization methods such as linear programming are used [14-17] to solve dispatch, optimal power flow and VAR optimization problems. Another powerful algorithm in this group is Mixed Integer Programming (MIP). This technique is used to optimize the operation of pump-storage hydro power stations, emissions and costs of thermal units, and scheduling of combined heat and power plant [18-20]. Dynamic programming is used to solve unit commitment problems [21]. Traditional optimization algorithms may not be as efficient as intelligence search methods when dealing with practical power system problems [22]. For instance, authors in [23] use the genetic algorithm to reduce the locational marginal price when solving the GR problem. In [2], generator sensitivities to the power flow on congested lines, i.e. the dependence of nodal power change to the power flow change, was discussed when dealing with GR by the PSO method.

In different countries and regions different congestion management methods are adopted. This paper considers a case study using the German power system, where only one wholesale price is allowed and low-cost generating units are concentrated in one particular region. Congestion management is realized using an optimal RD method. We assume a perfect competition in the market meaning that power plants submit their bids according to the marginal cost (i.e. quadratic cost functions are used). The optimization problem is solved using the PSO method, introduced in Section 2. With an increased number of renewable energy sources in a power system, the operation of traditional power plants still needs to be optimised based on physical limitations. These limitations (manoeuvrability, start up time, shut down time, etc.) are accounted for in the problem definition presented in Section 3. Section 4 elaborates on how the PSO algorithm is modified so that it can simultaneously minimize re-dispatch costs of each hour and each day resulting in an improved convergence. The case study is presented in Section 5. Conclusions are listed in Section 6.

\section{PARTICLE SWARM OPTIMIZATION}

The Particle Swarm Optimization method is a powerful and simple evolutionary algorithm developed at the end of the 20th century by Kennedy and Eberhart [25]. It was inspired by behaviour patterns of social communities (e.g. birds or fish) which search for food or better living conditions. It was recognised that some birds flying in the flock are not familiar with the exact position of their destination, however they know the location closest to it. Their strategy is to look for the destination around some area based on the cumulative knowledge.

In an optimization problem, each member of the community is represented by a particle. The community (comprised of these particles) is called a swarm. The position of each $i$-th particle denoted by $X_{i}=\left(x_{i 1}, x_{i 1}, \ldots, x_{i N}\right)$ is the first fundamental property of the $N$-dimensional particle and is a potential solution to the problem. A personal best denoted by $Y_{i}=\left(y_{i 1}, y_{i 1}, \ldots, y_{i N}\right)$ is the second fundamental property of a particle. Finally, $V_{i}=\left(v_{i 1}, v_{i 1}, \ldots, v_{i N}\right)$ is a velocity of the particle. The fundamental property of the swarm is called a global best $\left(\mathrm{G}=\left(g_{1}, g_{1}, \ldots, g_{N}\right)\right)$.

At the initial moment, the particle positions as well as their velocities are assigned randomly. As PSO is an evolutionary method, particles flying in the multidimensional space approach the optimum from iteration to iteration. At the end of each iteration, the particle positions are evaluated with respect to the objective function. With $n$ being the number of iteration and $f$ the objective function, the personal best of each particle is updated according to the following equation:

$$
Y_{i, n}= \begin{cases}X_{i, n} & \text { if } f\left(X_{i, n}\right)<f\left(Y_{i, n}\right) \\ Y_{i, n} & \text { otherwise }\end{cases}
$$

Particles share this information with other members of the swarm by updating the global best as:

$$
G_{i, n}= \begin{cases}Y_{i, n} & \text { if } f\left(Y_{i, n}\right)<f\left(G_{n-1}\right) \\ G_{n-1} & \text { otherwise }\end{cases}
$$

To get the new position, particles use scaled values of both personal and global best as shown in Figure 1 and Equations (3) and (4).

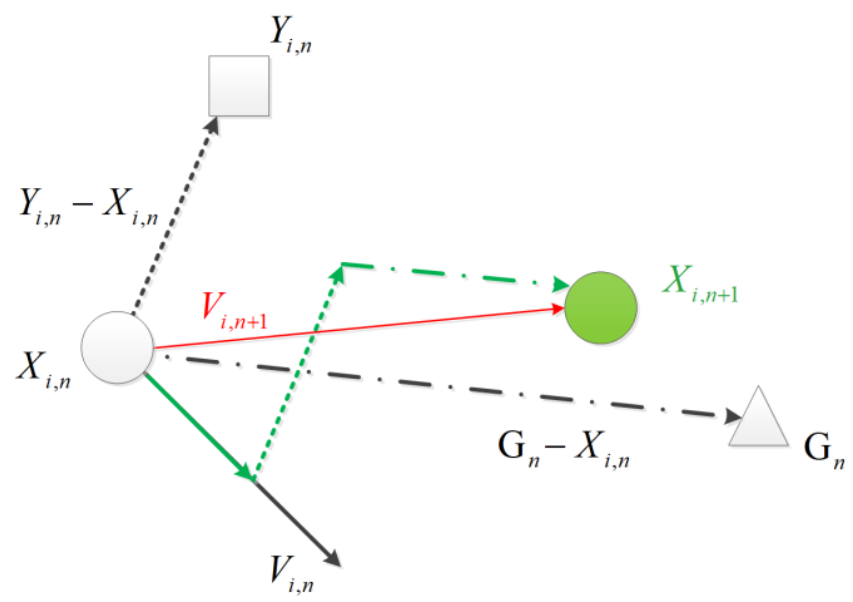

Figure 1. Visual representation of a PSO method.

$$
\begin{gathered}
V_{i, n+1}=w \times V_{i, n}+c_{1} \times r_{1} \times\left(Y_{i, n}-X_{i, n}\right)+ \\
c_{2} \times r_{2} \times\left(G_{n}-X_{i, n}\right) \\
X_{i, n+1}=X_{i, n}+V_{i, n+1}
\end{gathered}
$$

where $w$ is the inertia weight; $c_{1}$ and $c_{2}$ are acceleration coefficients; and $r_{1}$ and $r_{2}$ are uniformly distributed random numbers between 0 and 1 .

The main advantage of the PSO method over traditional optimization algorithms is that the objective function is not required to be differentiable and there is no need to predefine initial parameters. In addition, the method is able to avoid trapping into local solutions and can be parallelized. The main drawback of the PSO method is that optimization constraints are usually transformed into penalty functions and added to the objective function. Therefore, some optimum particle positions may be ignored which may result in bad convergence. This paper suggests an alternative technique for dealing with this problem. 


\section{CONGESTION MANAGEMENT}

\section{A. Sensitivity analysis}

In accordance with Kirchhoff's second Law, any change in the generated nodal power will entail power flows redistribution. However, every line has a different sensitivity to such changes. This phenomenon is accounted for by sensitivity analysis methods. Depending on the desired computational speed and accuracy, different approaches such as AC and DC load flow solutions (LFS), or AC and DC power transmission distribution factors (PTDF) are used.

This paper adopts the AC PTDF method, which is expressed by the following equation [3]:

$$
\left(D_{i-j}\right)_{m-n}=\frac{\Delta P_{i-j}}{\Delta T_{m-n}}
$$

where $D$ is the sensitivity (PTDF) matrix; $\Delta P_{i-j}$ the change in active power flow between nodes $i$ and $j$; and $\Delta T_{m-n}$ the change in transaction between $m$ and $n$.

\section{B. Dynamic constraints}

Electric power plants are operated in compliance with applicable codes and regulations [26]. Main constraints are the minimum and maximum power plant outputs depending on time $t$ :

$$
P_{G i, \min }<P_{G i}(t)<P_{G i, \max }
$$

The gradient of a power plant or its manoeuvrability is defined as the ability of the power plant to increase or decrease power output during a time increment $\Delta t$ :

$$
\begin{aligned}
& P_{G i}(t)-P_{G i}(t-1) \leq U R_{i} \text { for power increase } \\
& P_{G i}(t)-P_{G i}(t-1) \leq D R_{i} \text { for power decrease }
\end{aligned}
$$

Minimum up and down times describe how long a power plant has to remain in up state $\left(T_{i}^{o n}\right)$ and down state $\left(T_{i}^{\text {off }}\right)$ respectively. For example, a steam turbine has to be warmed up or cooled down when changing operating modes. Inequalities (9) and (10) express these phenomena.

$$
\begin{gathered}
{\left[X_{i}^{o n}(t-1)-T_{i}^{o n}\right] \times\left[I_{i}(t-1)-I_{i}(t)\right] \geq 0} \\
{\left[X_{i}^{o f f}(t-1)-T_{i}^{o f f}\right] \times\left[I_{i}(t)-I_{i}(t-1)\right] \geq 0}
\end{gathered}
$$

where $P_{G i}(t)$ is the power generated at node $i ; P_{G i, \min }$ and $P_{G i, \max }$ are the lower and upper limits of a power plant $i$; $U R_{i}$ and $D R_{i}$ the ramp up and down limits of the power plant $i ; X_{i}^{o n}$ and $X_{i}^{\text {off }}$ the duration of a power plant being online or offline before time $t$; and $I_{i}(t)$ the commitment status of unit $i$ at time $t$ (e.g. " 1 " or " 0 ").

\section{Problem formulation}

Objective function

$$
\min C=\min \sum_{t=t_{0}}^{N_{t}} \sum_{i=1}^{N_{G}}\left(\begin{array}{c}
I_{i}(t) \times b_{i} \times \Delta P_{G i}+ \\
+I_{i}(t) \times\left(1-I_{i}(t-1)\right) \times S U_{i}+ \\
+I_{i}(t-1) \times\left(1-I_{i}(t)\right) \times S D_{i}
\end{array}\right)
$$

where $b_{i}$ is the incremental or decremental price bids submitted by generators (in this paper for simplicity reasons $b_{i}=\left.\frac{d c_{i}\left(P_{G i}\right)}{d t}\right|_{P_{G i}^{0}}$ with $c_{i}$ being a power plant's cost function); $N_{t}$ is the considered time interval; $N_{G}$ the number of generating units participating in re-dispatch; and $S U_{i}$ and $S D_{i}$ the start-up and shut down costs.

System power balance

$$
\sum_{t=t_{0}}^{N_{t}} \sum_{i=1}^{N_{G}} \Delta P_{G i}(t)=0
$$

Security constraints

$$
\left|\sum_{i=1}^{N_{G}}\left(D_{i-j}\right)_{m-n} \times \Delta P_{G i}(t)\right| \leq \Delta P_{i-j \max }
$$

where $\Delta P_{i-j \max }=\left(P_{i-j \max }-P_{i-j}^{0}\right)$ is the incremental power flow limit and $P_{i-j}^{0}$ the power flow prior rescheduling.

Additionally, dynamic constraints of conventional generating units (6-10) presented in section $b$ are taken into account in the implemented model.

\section{CONGESTION MANAGEMENT USING PARTICLE SWARM OPTIMIZATION}

The optimization problem formulated in the previous section considers multiple time periods where the objective is to minimize the overall cost as well as the cost of each separate instance. In order to be able to solve this problem using particle swarm optimization, the method has to be modified. The particle's position $\left(X_{i}\right)$ is set to be equal to the incremental power in generating nodes $\left(\Delta P_{G i}\right)$. The swarm is subdivided into several families, each representing a single moment of time as shown in Figure 2.

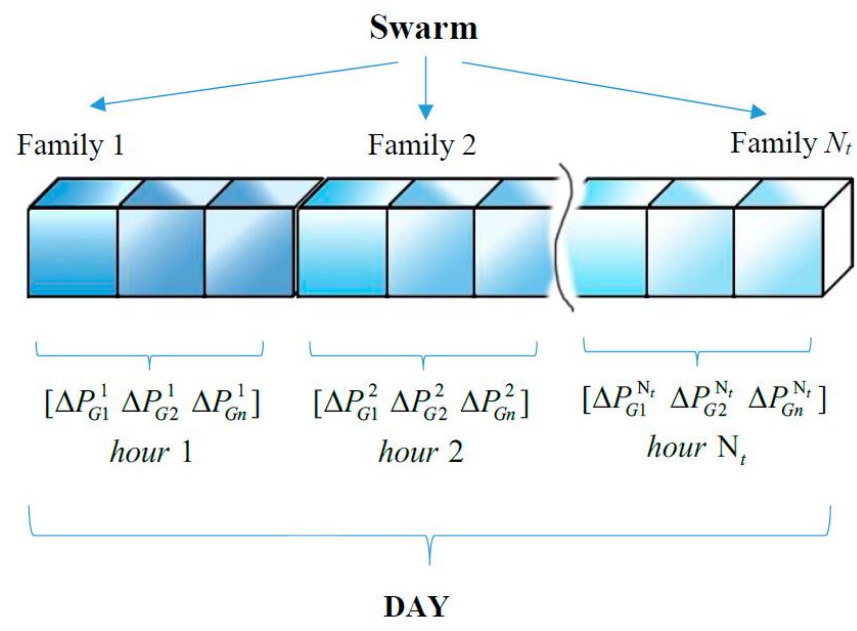

Figure 2. Hierarchy of a swarm.

The family's objective is to minimize the cost of redispatch both as an isolated unit and as part of a swarm. This can be achieved by replacing some fundamental properties of the traditional particle. As a result, a modified particle has two personal best positions, i.e. personal best as a member of the family $\left(Y_{i, n}^{\text {family }}\right)$ and as a member of $\operatorname{swarm}\left(Y_{i, n}^{\text {swarm }}\right)$. 
Global best of the swarm undergoes the same subdivision as shown in Figure 3.

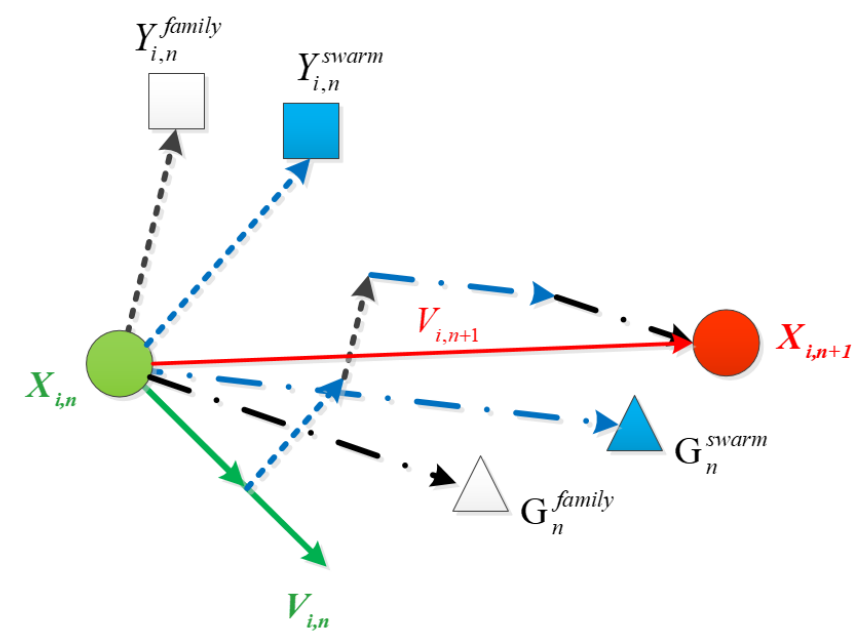

Figure 3. Visual representation of a modified PSO method.

Finally, Equations (3)-(4) can be replaced with (14)-(15) according to the proposed concept.

$$
\begin{gathered}
V_{i, n+1}^{*}=w \times V_{i, n}+c_{1} \times r_{1} \times \\
\times\left[c_{11} \times r_{11}\left(Y_{i, n}^{f}-X_{i, n}\right)+c_{12} \times r_{12}\left(Y_{i, n}^{s}-X_{i, n}\right)\right] \\
+c_{2} \times r_{2} \times\left[c_{11} \times r_{11}\left(G_{i, n}^{f}-X_{i, n}\right)+c_{12} \times r_{12}\left(G_{i, n}^{s}-X_{i, n}\right)\right] \\
X_{i, n+1}=X_{i, n}+V_{i, n+1}^{*}
\end{gathered}
$$

where $c_{11}$ and $c_{12}$ express how much the swarm's and the family's knowledge respectively influence the search.

\section{CASE STUDY}

A modified PSO method, explained in the previous section, was implemented in MATLAB and studied on the 8bus system shown in Figure 4.

The proposed network has one level of voltage $(110 \mathrm{kV})$, ten lines (for simplicity, lines have the same length and per unit parameters) and four generation units.

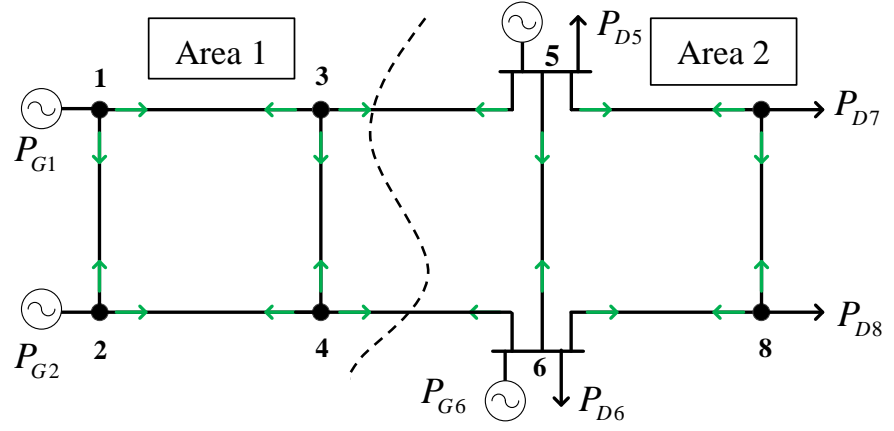

Figure 4. The 8-bus test network.

The network can be subdivided into two areas, which are area 1 with low cost generating capacity, and area 2 with high-cost generation and variable demand. Cost functions and constraints of generating units are presented in Table 1.

TABLE I. INPUT DATA

\begin{tabular}{|c|c|c|c|c|c|c|c|c|c|c|}
\hline \multirow{2}{*}{$\begin{array}{c}\text { Gen. } \\
\text { bus }\end{array}$} & \multicolumn{2}{|c|}{ Production Cost } & \multicolumn{2}{c|}{ Start-up cost } & \multicolumn{2}{c|}{$\begin{array}{c}\text { Ramp } \\
\text { limits }\end{array}$} & \multicolumn{2}{c|}{$\begin{array}{c}\text { Time } \\
\text { limits }\end{array}$} \\
\cline { 2 - 12 } & $\boldsymbol{a}$ & $\boldsymbol{b}$ & $\boldsymbol{c}$ & $\boldsymbol{\alpha}$ & $\boldsymbol{\beta}$ & $\boldsymbol{\tau}$ & $\boldsymbol{U} \boldsymbol{R}_{\boldsymbol{i}}$ & $\boldsymbol{D} \boldsymbol{R}_{\boldsymbol{i}}$ & $\boldsymbol{o n}$ & $\boldsymbol{o f f}$ \\
\hline 1 & 0.006 & 7.3 & 500 & 1460 & 650 & 3 & 55 & 55 & 2 & 2 \\
\hline 2 & 0.003 & 5.1 & 200 & 2100 & 950 & 4 & 55 & 55 & 2 & 2 \\
\hline 5 & 0.009 & 10.5 & 50 & 2100 & 950 & 5 & 15 & 15 & 2 & 2 \\
\hline 6 & 0.008 & 8.5 & 10 & 1180 & 625 & 2 & 15 & 15 & 2 & 2 \\
\hline
\end{tabular}

Due to the change of demand, lines 1-5 and 2-6 became overloaded, as shown at the bottom of Figure 5. Generating unit (Gen 6) with the highest cost of production in the second area had to be re-dispatched down, whereas the power plant (Gen 1) with the lowest power cost in area 1 was redispatched up, thus clearing system congestion.

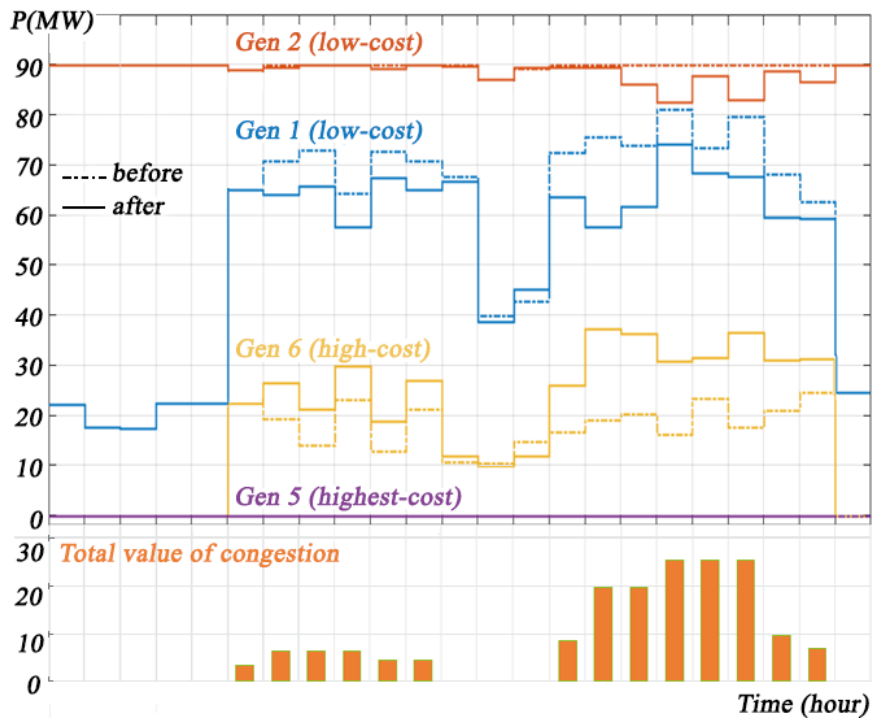

Figure 5. Rescheduling due to line overloads.

In order to find the optimum solution to the problem, several independent swarms are used with the different velocity coefficients values as shown in table 2 .

TABLE II. VELOCITY COEFFICIENTS

\begin{tabular}{|c|c|c|c|c|c|c|}
\hline Swarm & $w$ & $w_{\text {damp }}$ & $\boldsymbol{c}_{\boldsymbol{1}}$ & $\boldsymbol{c}_{\boldsymbol{2}}$ & $\boldsymbol{c}_{\boldsymbol{1 1}}$ & $\boldsymbol{c}_{\boldsymbol{1} \mathbf{2}}$ \\
\hline 1 & 1 & 0.99 & 2 & 2 & 0.1 & 0.9 \\
\hline 2 & 0.5 & 0.99 & 1 & 2 & 0.1 & 0.9 \\
\hline 3 & 0.5 & 0.99 & 0.5 & 2.5 & 0.1 & 0.9 \\
\hline
\end{tabular}

Swarms of type 2 and 3 yield more stable results and have better convergence, as depicted in Figure 6. 


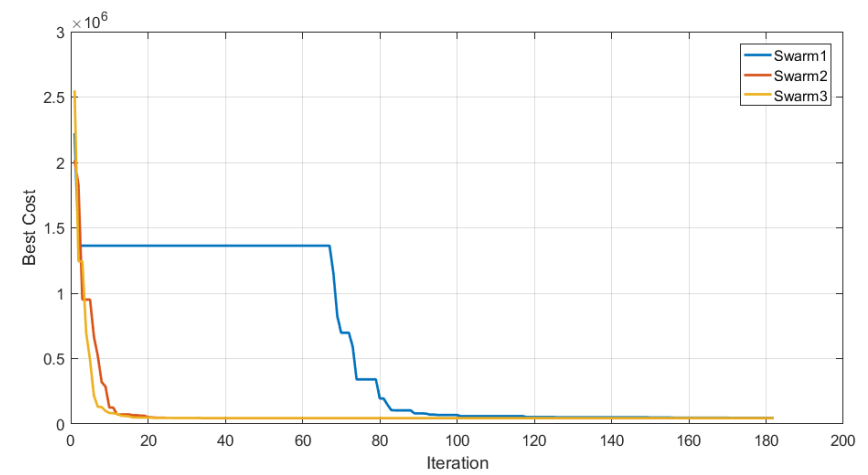

Figure 6. Convergence of PSO method with several swarms.

\section{CONCLUSION}

The paper presents a congestion management methodology for deregulated electric power systems with a large share of renewable energy sources. In order to address congestion, the optimal generator rescheduling problem considering dynamic limitations of conventional power plants was solved using the particle swarm optimization method. In order to improve the ability of the method to satisfy multipletime objectives several adjustments are proposed. The swarm with its global goal is subdivided into several families each having their own objectives. The suggested technique was tested on the 8-bus power system divided into two areas with low and high costs of generation. Optimal rescheduling of conventional power plants considering their manoeuvrability, start-up and shut-down times is obtained, resulting in a congestion free power system.

\section{ACKNOWLEDGMENT}

The authors acknowledge the contributions of Christian Klabunde from Otto-von-Guericke University Magdeburg in the model development, and Zane Smith from University of Tasmania for proof-reading the paper.

\section{REFERENCES}

[1] F. Flores-Espino, T. Tian, I. Chernyakhovskiy, and M. Mercer, "Competitive Electricity Market Regulation in the United States: A Primer," National Renewable Energy Lab., Denver, CO, Tech. Rep. NREL/TP-6A20-67106, Dec. 2016.

[2] Ariette Nuessler, "Congestion and redispatch in Germany. A modelbased analysis of the development of redispatch", Ph.D. dissertation, Inst. of Energy Econom., Univ. of Cologne, Cologne, 2012.

[3] N.D.Ghawghawe, and K.L.Thakre "Application of Power Flow Sensitivity Analysis and PTDF for Determination of ATC," in Proc. 2007 IEEE PEDES

[4] Mun-Kyeom Kim ; Dong-Hyeon Kim ; Yong Tae Yoon ; Sang-Seung Lee ; Jong-Keun Park "Determination of Available Transfer Capability Using Continuation Power Flow with Fuzzy Set Theory," in Proc. 2007 IEEE Power Engineering Society General Meeting.

[5] Y. Ou ; C. Singh "Assessment of available transfer capability and margins," in Proc. IEEE Transactions on Power Systems (Volume: 17. Issue: 2, May 2002), pp. $463-468$.

[6] A. Yousefi, T. T. Nguyen, H. Zareipour, O. P. Malik, "Congestion management using demand response and facts devices", International
Journal of Electrical Power \& Energy Systems, vol. 37, no. 1, pp. 78$85,2012$.

[7] K. Mwanza, Y. Shi, "Congestion management: re-dispatch and application of facts," Ph.D. dissertation, Dep. of Energy and Environment, Goteborg, Sweden, Chalmers Univ. of Technology, 2006.

[8] A. Swami, "Transmission Congestion Impacts on Electricity Market: An overview," Proc. in IJETAE, vol. 3, 2013.

[9] Emily B. Fisher, Richard P. O’Neill, Michael C. Ferris "Optimal Transmission Switching" IEEE transactions on power systems, VOL. 23, NO. 3, AUGUST 2008

[10] G. Granelli, M. Montagna, F. Zanellini, P. Bresesti, R. Vailati, and M. Innorta, "Optimal network reconfiguration for congestion management by deterministic and genetic algorithms," in Proc. Elect. Power Syst. Res., vol.76, pp. 549-556, 2006.

[11] Scott M. Harvey, William W. "Nodal and Zonal Congestion Management and the Exercise of Market Power," Harvard University, 2000.

[12] Daniel Kirschen, Goran Strbac "Fundamentals of Power System Economics", John Wiley \& Sons Ltd, UK 2004.

[13] Nurul Idayu Yusoff ; Abdullah Asuhaimi Mohd Zin ; Azhar Bin Khairuddin, "Congestion management in power system: A review," in Proc. Of 2017 3rd International Conference on Power Generation Systems and Renewable Energy Technologies (PGSRET), Johor Bahru, Malaysia, pp. 22-26.

[14] Said Ahmed-Zaid "Optimal load flows using linear programing" 1980.

[15] O. Alsac, J. Bright, M. Prais, and B. Scott. "Further Developments in LP Based Optimal Power Flow.” IEEE Trans. Power Systems, 5(3), pp. 697-711, 1990.

[16] G.D. Irrisari, L.M. Kimball, et al. "Economic Dispatch with Network and Ramping Constraints via Interior Point Methods." IEEE Trans. Power Systems, 13, 236-242, 1998.

[17] I.A. Farhat M.E. El-Hawary "Interior point methods application in optimum operational scheduling of electric power systems", IET Gener. Transm. Distrib., Vol. 3, Iss. 11, pp. 1020-1029, 2009

[18] Mohammad RezaNorouzi , AbdollahAhmadi, et al., "Mixed integer programming of multi-objective security-constrained hydro/thermal unit commitment", Renewable and Sustainable Energy Reviews 29, pp. 911-923, 2014.

[19] Alberto Borghetti, Claudia D'Ambrosio, Andrea Lodi, Silvano Martello, "An MILP Approach for Short-Term Hydro Scheduling and Unit Commitment With Head-Dependent Reservoir." IEEE transactions on power systems, vol. 23, no. 3, 2008 .

[20] Jong Suk Kim, Thomas F. Edgar, "Optimal scheduling of combined heat and power plants using mixed-integer nonlinear programming." Energy, 77, pp. 675-690, 2014.

[21] S.Usha Rania, C. H. Padmanabha Rajua, "A Solution to Unit Commitment Problem via Dynamic Programming and Particle Swarm Optimization" International Journal of Current Engineering and Technology, Vol.3, No.4, 2013.

[22] Soliman Abdel-Hady Soliman, Abdel-Aal Hassan Mantawy "Modern Optimization Techniques with Applications in Electric Power Systems", Springer Science 2012.

[23] A. Eladl, S. Kaddah, E. Haikal, "Optimal Generation Rescheduling for Congestion Management, LMP Enhancement and Social Welfare Maximization," in Proc. Of 2017 Nineteenth International Middle East Power Systems Conference (MEPCON), Menoufia University, Egypt, pp. 1190-1194.

[24] S. Dutta and S. Singh, "Optimal rescheduling of generators for congestion management based on particle swarm optimization," in Proc. IEEE Trans. Power System., vol. 23, pp. 1560-1569, 2008.

[25] A. Kennedy and R. Eberhart, "Particle Swarm Optimization," in Proc. IEEE Int. Conf. Neural Networks, Nov. 29-Dec. 1 1995, vol. IV, pp. 1942-1948.

[26] C. Wang, S. M. Shahidehpour, "Effects of ramp-rate limits on unit commitment and economic dispatch", Proc. in 1993 IEEE Transactions on Power Systems, Vol. 8, No. 3. pp. 1341-1350. 